\title{
Enabling Positive Practice Improvement through Data-Driven Growth: A model for understanding how data and self-perception lead to practice change
}

\author{
Rana Kamhawy ${ }^{1}$, Teresa Chan ${ }^{1}$, and Shawn Mondoux ${ }^{1}$ \\ ${ }^{1}$ McMaster University
}

June 26, 2020

\begin{abstract}
Purpose This paper aims to elucidate the factors that play into physicians' experience of receiving practice data and to subsequently develop a model that describes how individuals may interact with the data they receive. Methods In a prior study, we conducted a needs analysis of 105 physicians in the Hamilton-Niagara area in order to understand which data metrics were most valuable to physicians. Using these results, we designed an interview guide to study physicians' perspectives on audit and feedback. By intentional sampling, we recruited 15 physicians amongst gender groups, types of practice (academic vs community), and duration of practice. The interviews were conducted by a single author and transcribed without identifiers. We then began with an open coding analysis for all of the transcripts, and thereafter conducted axial coding to group the data into larger themes. Results Several environmental and personal attributes were identified as either enabling or counterproductive attributes for participant improvement. The final proposed model identifies different zones of engagement on the basis of both the individual practitioner's growth mindset and the quality of the existing data system. In the highest engagement zone, the mindset of the collective leadership is one of growth. Systemic supports are in place which potentiates learning that may come from an individual motivated to use their own data. Conclusion Our model shows how data feedback systems and individual growth-oriented mindsets interact to augment or hinder clinical practice improvement. This model provides important guidance to academic and administrative structures looking to develop appropriate performance feedback systems with clinicians.
\end{abstract}

\section{Introduction}

In an effort to improve clinical care and provide more quantitative feedback to clinicians, the practice of audit and feedback (A\&F) has been implemented as a quality improvement (QI) initiative in many clinical practice settings ${ }^{1}$. Systematic reviews have demonstrated that the net effect of these interventions on overall performance is small ${ }^{1}$ and that implementation for selected measures is done ineffectively ${ }^{2}$. While the literature on the implementation of best practices for $\mathrm{A} \& \mathrm{~F}$ measures is increasing ${ }^{3}$, the endpoints of clinical practice change for end-users (physicians) is rarely assessed.

At present, there is very little guidance on how to use a refined A\&F tool that has been well implemented to the minds, hearts and behaviors of physicians working in the clinical space. In other words, how do we take what is known in the field of $\mathrm{A} \& \mathrm{~F}$ and translate this into meaningful practice change for practitioners? Many fields are currently asking the same question. The proliferation of discussions around clinical coaching ${ }^{4}$, expanded continuing professional development (CPD) curricula, the inclusion of design thinking modalities and the creation of peer learning spaces ${ }^{5-8}$ are all indicative of a variety of "specialties" looking to answer the same question.

In response, we sought to develop an understanding of how physicians experienced the act of receiving practice data and develop a model that describes how individuals may interact with the practice data that 
they receive. Such a model would guide a series of different interventions across a variety of data and "readiness to change" environments. It may also provide insights on how best to design data systems and how to approach clinicians for change improvement within different data and change contexts.

\section{Methods}

In this study, we used constructivist grounded theory ${ }^{9}$ to explore how physicians interacted and reacted to a variety of data elements presented in ED audit and feedback. This project is the second in a multiphase needs assessment aimed at developing a new approach to A\&F. In prior work, we conducted a regional needs assessment for A\&F for emergency physicians, soliciting which practice data elements were most desired regarding practice measurement. ${ }^{10}$ The results of this regional needs assessment were stratified into the subgroups of respondents. These were divided by gender, duration of practice, and type of practice (community vs academic). By asking physicians to provide their own reactions as well as their perceptions on differences we observed between subgroups, we looked to gain insight into how they may react to their own practice data.

Reflexivity. Before engaging in any analyses, our investigatory team met to discuss our inherent stances and assumptions in order to assist us in achieving reflexivity. Each member of the analysis team sought to declare their perceptions about the role and value of $A \& F$ and the predicted view of $A \& F$ by the participating subgroups. We also discussed our prior training (if we had any), and the sensitizing concepts/frameworks brought to the analysis process. The research team then made a plan for the theme extraction of interviews.

Population/Recruitment. A total of 15 participants were recruited for interview participation. Intentional sampling was performed to ensure that participants represented a variety of practice characteristics that were identified in the needs assessment (gender, community vs. academic practice, and duration of independent practice). Participants were emergency physicians practicing in the Hamilton region amongst 7 practice sites which were included in our needs analysis. All interviews were conducted between July $15^{\text {th }}$ and September $2^{\text {nd }}, 2019$.

Interview Procedure. The interview guide was constructed by a single author (SM) based on the needs assessment survey data. This was refined and finalized by a second author with significant qualitative research experience (TC). Interviews were conducted by our first author (RK) after undergoing training with the more experienced members (TC, SM) of the team and receiving feedback. A training interview was conducted with this author before the inclusion of the research subjects. Impressions and insights from participants were collected during a single phase of interviews with all participants. Interview scripts are available in Appendix A.

Ethical review. $T$ his project received an ethical exemption for the Hamilton Integrated Research Ethics Board (HiREB).

Analysis. I nterviews were audio-recorded and then transcribed without identifiers. The author who interviewed participants was the only author who was privy to the identity of the respondents. We analyzed the data using a constant comparative method to identify these, initially starting with an open coding process for all of the transcripts. Once this was complete, we proceeded with axial coding to group relevant themes and subthemes.

For the first transcript, the entire research team met to normalize the process of open coding for all. Thereafter, two authors (SM, RK) met and coded all the subsequent transcripts. With each analysis meeting, codes were discussed, added, or collapsed our previous coding. Our team opted to resolve disagreements through a discussion and consensus-building process. We eventually came to a consensus on all themes presented in our final analysis. Two authors subsequently met (SM, TC) for the axial coding of the data into larger themes.

Methods to Increase Rigor. As a form of member check, we sent the penultimate version of our newly developed model to our participants for their review and comment. We asked them to review the figure and 
associated explanations and provide insights about whether this model suited their worldview. Amendments and questions were used to revise the model to generate our final version.

\section{Results}

A total of 15 interviews were conducted and transcribed of which 6/15 (40\%) were female, 6/15 (40\%) had an exclusive community practice and the range of duration of practice extended from 2 to 30 years with an average of 11.3 years. The features of each participant are found in Table 1. The duration of interviews ranged between 33 minutes to 70 minutes.

(table 1)

\section{Enabling Attributes}

Self-improvement culture of the practice site: Academic vs. Community sites

Enabling elements centered on the improvement culture of the practice site were largely elicited when respondents were asked to compare academic and community practices. Many stated that improvement and practice measurement were expected to a higher degree in academic settings. One participant [P4] commented, "... In an academic setting you are just geared to continuous education, continuous learning, continuous improvement."

In addition, some speculated that this was the result of more academic training programs and credentialing. Respondents remarked that a personal history of taking part in several elements of advanced training may drive an increased reliance on quantitative metrics. Participant 7 explained:

"I think that there is just this kind of more of an expectation that people in academic facilities to be measured then what would be in a community hospital."

Another element elicited when comparing community vs academic sites was the perceived focus on physician performance. Some respondents suggested that academic physicians were more driven to understand their performance relative to their peers and therefore respond to practice data for improvement. Some believed that the presence of performance metrics did or could drive competition within metrics that may be more socially valued.

"And that is why I think some people prefer to practice in academic settings rather than community it is because they are more performance oriented. They think that they are doing well but they always want to be I guess challenged and driven in this way." [P11]

Lastly, some respondents stated that self-improvement was most essential in academic facilities due to their role as educational centers for residents and medical students. They suggested that self-improvement may be driven by the need to act as the best possible clinical role model for learners.

"You are teaching, you have learners. So, you have to set an example to those learners, and you have to be able to teach them and prepare them for their careers. So, you want to improve yourself first before you can do all of that." [P9]

\section{Individual engagement and a broader culture of engagement}

A broader sense of community in the delivery of healthcare as well as in establishing the values of care were common when discussing the culture of engagement. Some practitioners identified that the sense of community and teamwork found in smaller non-academic settings was an enabler, although academic practitioners also identified that a sense of common values was important in enabling a culture of practice change.

"I think [...] they see themselves as a component of a larger team that's goal is to provide the best care for patients." [P4] "The usual people that care deeply about others and have a team mentality I think (would take part) as long as they felt that the metric was valid, and it was an accurate reflection of (practice)." [P5] 
Community providers also noted that their engagement in positive patient outcomes may be derived from their individual and departmental relationships with patients and the community rather than performance. This sense of a broader community was suggested as an enabler of engagement and practice change

"You know everyone better and you may know some of the patients and the staff that you are working with more on a personal level then you would in a larger institution that is more formal and more rigid." [P11]

Existence of self-data feedback loops

Many respondents reflected on how the data would make them feel about themselves and how it might drive them to change their behavior or practice. Many endorsed a level of curiosity about quantitative measures of their practice- some of which supported the use of data for validation of good practices. Some physicians acknowledged that some positive data would be attributed to team performance rather than individual physician performance.

"Maybe they already know that they are doing a great job and they just want to be validated some more." [P8] "So good practice feedback. I think that would be reaffirming for them but also, they may kind of shrug it off as I am just a part of the team." [P4]

Others raised important insights into the consequences of data feedback that may be viewed as negative by the recipient. They highlighted that the risk of these could be inappropriate remedial actions to improve data metrics or that the data may be seen as a value judgement on their character. They also stated that these may conflict with their own image of themselves, forcing some reflection.

"Yeah so, I think that they would um, see it as a value judgement on their character. And so, may not be as open if it is not congruent with their philosophy on how (they are) part of the team. And also, how they are for this overall community of the emergency department." [P7]

While recognizing this potential, many respondents reflected that the act of acquiring practice data would lead to an ongoing assessment of personal practice as well as the identification of areas where there is a large potential for improvement. This could lead to a deeper understanding of negative performance in these clinical areas.

"And so, I think [..] physicians recognize that there are always going to be areas in their practice that you know have a broad ability to improve" [P5] " And we might imagine that the utility will be less because we I think probably mistakenly assume that we have little to learn. When in fact my guess is probably that we can learn a lot." [P15]

In many cases, there was a clear focus on elements of quality of care rather than simply throughput metrics, which represent a thoughtful prioritization of A\&F metrics. Personal improvement loops centered on elements of quality of care are enabling attributes in $\mathrm{A} \& \mathrm{~F}$.

"[..] or maybe they value more the spending time and communicating better with their patients then the just sheer volume of patients seen per shift. ... maybe they value you know better care for patient care than volume. And that is a different way to look at things." [P2]

\section{Counterproductive Attributes}

Questioning data validity

Questioning data validity occurred during the discussion of many areas of data feedback although were particularly relevant in questions centering around patient satisfaction and teaching evaluations by learners. Many of the physicians' interviews mentioned that, despite seeing the need for measuring patient experience and satisfaction, the importance placed on this metric by the system and patient were likely to be more significant than their own assessment of importance and could, in some instances, be driven by social factors.

"We have a very high proportion of patients who are under housed, with mental health and addictions and so, there might be a signal there that the reliability of patient data on the quality of care may be influenced 
by the patient population [...]." [P15]

Several clinicians commented on the uniqueness of data reporting in a community setting, reducing in many cases its validity. Some stated that the nature of community EDs rendered data reporting less useful as outcomes may already be known for patients returning to the ED. Patients may be returning for planned visits or to see their primary care physician in the ED.

"So, they might think that numbers might get skewed. So, in the community there might be a lot more bring back patients for things like imaging tests, [intravenous] antibiotics etc., so those numbers may get skewed more in the community because there are more bring back tasks." [P6]

Some female respondents questioned the validity of the data by stating that the metrics currently being measured often do not resonate with female practitioners. They also stated that those measures in which female MDs excel are not often part of the datasets measured by departments or that the data metrics measured were not part of their personal values.

"But if the simple metric is patients per hour then there might be gender affect there that could get interpreted in the wrong way which would say that women should work faster." [P15] "I think that they would place little value in that. I think that they may see it as incongruent with their own philosophy of how they practice." [P7]

\section{"Data can't change me!"}

Many of the conversations grouped in this category were achieved using a gender lens of practice in the ED. Most of the responses generated in these discussions centered on the incongruence of values between genders. Gender inequity in clinical practice was not explicitly explored as part of this work, only reactions to data from each gender subgroup. Although many of the discussions center on gender themes, the logic used to discredit a data metric was seen across all subgroups of participating respondents.

Female respondents demonstrated a significant aversion to throughput metrics. Themes were based on sentiments of higher clinical scrutiny for female practitioners as well as reduced value placed on throughput measures.

"We are also way more cautious and way more scrutinized than our male colleagues are." [P14] "I would say less women are driven so much by the money aspect of it than the guys are. So, the guys are usually much more driven by how many patients they see and what they bill etc.. And that is not entirely true for everybody but whereas women seem to be less drawn into it." [P10]

Instead, female practitioners stated they placed more emphasis on metrics related to the quality of patient care than those related to throughput, an element otherwise seen as a positive attribute previously quoted as an enabling feature. The aspect of this feedback which is considered counterproductive is the outright rejection of a practice metric in favor of another, a logic which could be broadly applied throughout the range of metrics. This was also seen in a subset of academic clinicians, who stated that adherence to practice guidelines was more important than patient satisfaction, therefore minimizing the patient experience.

"So, probably, women value the quality of care delivered to the patients maybe or maybe they value more the spending time and communicating better with their patients then the just sheer volume of patients seen per shift... [M]aybe they value you know better care for patient care than volume. And that is a different way to look at things." [P2]

Some clinicians viewed data exercises such as audit and feedback as attempts at practice standardization, leading to the belief that this would remove physician autonomy. The views expressed within this section of the manuscript raised important questions about our expectations of care and practice uniformity.

"... if it becomes that this is secretly a process to make sure that I act and behave like an automaton then it becomes a real problem because physicians are wired appropriately or perhaps inappropriately to really value that autonomy." $[\mathrm{P} 15]$ 


\section{The feeling of being overwhelmed or helpless}

Many clinicians exhibited feelings of being overwhelmed with the data or feeling helpless as a result of receiving data. Many explicitly endorsed a devaluation of self-image and stated that it could be difficult to face their perceived shortcomings before moving to an exploration of practice improvement. Some respondents identified that it may be easier to not be made aware of opportunities for improvement.

"I think somebody like that who already thinks that they are giving their all and being pretty egalitarian $[\ldots]$, they would probably take it very to heart and be hurt by it. And a little defensive." [P8]

"I think because they don't want to be judged. Or maybe they don't want others to think that they are not good clinicians. Maybe that is why. Or maybe some people have a hard time facing their mistakes or their weaknesses and they don't want to know about them." [P9]

Disengagement with the purpose of data

Many clinicians were disengaged with the purpose of providing data and engaging in A\&F activities. Through an academic lens, some stated that measuring adherence to practice standards through audit and feedback may represent a complex to identify a "minimum standard" which should be occurring in the clinical setting. Some respondents also identified that certain remunerative environments may drive physicians to care more or less about A\&F data.

"Well there are a couple things, one is that it depends on how you're funded so if you're salaried then it doesn't really matter how many you see. So, it really depends on [...] if you are salaried or fee for service." [P14]

Through a gender lens, some female practitioners stated that they felt that emergency medicine was a male dominated environment with largely male leadership, leading to measurements and improvement targets that may not resonate with women.

"Yeah, I think that it is a very male dominated environment. And most of the staff are men, and the higher-ups are older white men. And there is a very buddy buddy system.' [P8]

Most respondents identified that participation in A\&F must occur under the pretext of being compatible to measurements established by the practice group and department. If a practitioner feels they are "on the outside" of the clinical group, their identification with the A\&F data would be less important.

"And you know sometimes what that leads to "is this isn't the right department for me", but the question is does somebody want to keep working in a department that isn't respectful of them." [P3]

\section{A New Model: Data-Driven Growth Spectrum}

Based on our findings and the various themes that we isolated from our data, we propose a singular model that articulates how each of these elements intersect to foster or prevent the acceptance or usage of A\&F procedures.This is dubbed the MD-PIE model (Mindset and Data model for Practice Improvement and Engagement). See Figure 1 which details the various elements.

Sensitized by several known frameworks including Carol Dweck's growth mindset framework ${ }^{11}$ and Kegan \& Lahey's Deliberately Developmental Organizations ${ }^{13}$, we developed a framework that integrates both the enablers and barriers to A\&F adoption that we had previously described.

(figure 1)

The proposed MD-PIE model describes the interactions of data systems and practitioner mindset in their ability to interact with and extract learnings from their practice data. Our interviews support the notion that some practitioners will wholeheartedly reject all practice data, specifically in those areas that identify opportunities for improvement, by calling into question the validity of the data. This is a ubiquitous reaction 
to the provision of practice data, yet some practitioners are able to move beyond the stage of data resistance into areas of pre-contemplation or change.

The helplessness and disengagement zones identify those practitioners that acknowledge the value and conclusions that come from the data yet do not feel empowered or are unsure how to move forward with the data that is available. Practitioners may move out of this area in two distinct ways. The first is by increasing the data quality that is coming to these individuals, notably by augmenting observational data with clear action plans for improvement and change which are based on the practice data. The second, which may be more difficult, would be to develop a growth mindset within the individual. Our interviews indicate that this may be easier in early career individuals.

Practitioners in the self-data feedback loop are already using the practice data to critically observe their own practice and make determinations on how they may improve. This may be achieved by individuals with a growth mindset, even in the context of low-quality data systems. Some individuals with more fixed mindsets may achieve this outcome with data systems of increased robustness which may, in some contexts, provide action plans for individual improvement around specific data elements. It should be noted that individuals within this category are augmenting their knowledge and practice mostly through self-reflection and selfmediated methods. Rarely do they encompass the efforts of the larger groups or use collective methodologies to identify opportunities or solutions. In other words, they do not leverage the collective wisdom which surrounds specific practice metrics.

The systemic supports zone is an area where the mindset of the collective leadership is a growth mindset. It places systemic supports in place which potentiate and augment the learning that may come from a single individual looking into their own data. Good examples of this include the literature around $\mathrm{R} 2 \mathrm{C} 2{ }^{7}$, which proposes the creation of small peer groups for the discussion of individual practice with the goal of improvement. Others could include specific coaching constructs around certain elements of clinical practice. Beyond this, some may include short, mid and long-term improvement targets as social contracts with peers. Clinicians were most comfortable engaging in A\&F activities within climates of systemic support for improvement.

\section{Discussion \& Implications of the MD-PIE model}

Our model is complementary to the $\mathrm{R} 2 \mathrm{C} 2$ model and would be augmented by the application of a similar standardized feedback modality ${ }^{5}$. The $\mathrm{R} 2 \mathrm{C} 2$ model provides a schema for feedback interaction and allows for the exploration of understanding of the clinicians. The "reaction" portion of this model is where most of our model would reside. Our model would provide perspective to the interviewer on where most physicians will fit, relative to the individual practitioner's mindset and the quality of the data system. Our model would then provide the interviewer with an opportunity to map a method by which an individual could come to reflect on the content of the feedback with an opportunity to grow, feeding directly into the Content portion of $\mathrm{R} 2 \mathrm{C} 2$. Our model may also identify opportunities for improvement of the data presented in feedback, allowing for iterative change and improvement on the data used for $\mathrm{R} 2 \mathrm{C} 2$. This reliance on a robust data model is not explicitly discussed in the $\mathrm{R} 2 \mathrm{C} 2$ framework.

When compared to other group feedback work using $\mathrm{R} 2 \mathrm{C} 2^{7,8}$, our model places similar emphasis on high quality data to enable appropriate discussion. The CAFF model provides outstanding direction for practitioners who have some modicum of interest in professional feedback loops relative to their own practice data. Our model describes how these interactions might work across many practitioners of a variety of growth mindsets. Our model also describes how robust data may contribute to bringing individual practitioners from helplessness to engaging with self-data feedback loops and allowing for more robust discussion in the Content and Coach areas of the $\mathrm{R} 2 \mathrm{C} 2$ model.

When comparing our model to Kegan and Lahey's Immunity to Change (ITC) model ${ }^{12}$, we find that these reconcile very well. Their published works on assessments for competing commitments should be performed most readily within individuals found in the helplessness and disengagement territory. There is also a portion of individuals in the data resistance category that may be choosing not to engage secondary to competing 
commitments. Those in the data invalidity category may receive a staggered approach to involvement; first, by understanding their suggestions for the improvement of practice measurement and implementing some change where warranted and second, by subsequently considering the application of the ITC framework if change remains difficult.

Lastly, a comparison of our model to the Deliberately Developmental Organizations (DDO) framework, also by Kegan and Lahey ${ }^{13}$, was warranted. DDOs operate under the inherent belief that investing in the development of each practitioner within the organization is in the best interest of the organization's own development and growth. This speaks to an overall mindset of improvement and adaptation to the current practice environment. The application of the DDO model to our framework would likely shrink the data validity and resistance areas and push more individuals into the systemic support region, whereby the department itself has a process in place to ensure individual improvement for all of its members. A DDO organization would seek to hire and push everybody into the systemic supports area.

This model provides a rubric through which academics, researchers and administrators may understand the interplay between an individual's growth mindset and the data systems providing quantitative practice feedback. The model consists of a "population-level" framework which should be applied to physician groups. Within our interview cohort, important themes were raised that reflected the perspectives of specific subgroups which could provide a more nuanced approach to data discussions and improvement. These nuances were not explicitly included in the model as they likely require additional research.

This also provides some guidance with the operational deployment of audit and feedback frameworks as it informs those conducting the implementation to expect a population who will resist this change throughout yet also reinforces the need for robust data systems to augment and enable improvement. This also allows administrators to integrate the concepts of early adopters, bystanders and nay-sayers ${ }^{8}$ with the data systems that may enable downstream improvement across categories.

Discussion on expectations of practice uniformity

In discussing $\mathrm{A} \& \mathrm{~F}$, our work shows that there may be individual reluctance to engage with data due to perceived risk on physician autonomy and expectations for increased uniformity. This highlights both the need for appropriate metrics and performance comparisons. In using evidence-based and patient-relevant metrics, physicians may come to see the utility of performance measures beyond simply standardizing clinical decisions. Specifically, using metrics for which there is a gold standard has previously been shown to have better feedback acceptance. Prior work by Gude et. al ${ }^{14}$ also recommends using tailored performance benchmarks, as opposed to standardizing against the group mean, to make the metric relevant and comparable. Individualized feedback thus becomes an important part of the feedback cycle to help recipients set personal targets rather than seeing metrics as an externally imposed performance comparator.

Finally, our model defines the various groups that may exist along the spectrum of data engagement. There are important considerations with regards to how these groups interact within one department. The Calgary Audit and Feedback Framework ${ }^{7}$, stemming from the Social Learning Theory, emphasizes that efficient learning can occur through reflecting, sharing practices, and discussing best evidence within the group. Based on our model, we anticipate that a department with individuals who prioritize audit and feedback can positively influence others to reflect on improving their practice in the setting of best evidence.

\section{Limitations}

Our study design inherently has selection bias due to intentional sampling. In addition, our study was limited to the Hamilton-Niagara region and may not encompass the experience of more rural Emergency Department settings. Finally, there is a smaller representation among mid-career physicians (6-15 years into practice) compared to those in early or later stages. This may be problematic because this group likely has the most refined practice patterns, and may have been a minority opinion that may have swayed our data one way or another.

\section{Future Directions}


Several complementary areas of research would augment these findings. Among those concepts requiring a deeper dive are the variation in values in performance metric measurement between genders and practitioners at different stages of clinical practice. This understanding could lead to the application of specific metrics within these populations or guide more effective discussions. They may also expose important biases that are embedded in specific measurement strategies and metrics. Lastly, they may call into question the equitability of the process to determine which metrics will be used to assess performance.

In addition to variability from within the interview cohort, our work had led us to consider whether excellent performance should be defined in a single manner from within a clinical group, academic structure or administrative construct. There are likely elements of performance that are ubiquitous to all physicians (elements such as safety and patient reported outcome measures) and others which may be less universal. This concept gets to the notion of whether we ask all physicians to change to move to a specific phenotype of practitioner or whether we accept a variety of clinical phenotypes and define good clinical practice (including performance variables) for each of these.

\section{Conclusion}

We present a model for understanding how high-quality data feedback systems and individual growth-oriented mindsets interact to augment or detract from clinical practice improvement. This model provides important guidance to academic and administrative structures looking to develop appropriate performance feedback systems with clinicians. This is foundational work in physician practice improvement.

\section{References:}

1. Ivers N, Jamtvedt G, Flottorp S, et al. Audit and feedback: effects on professional practice and healthcare outcomes. Cochrane database Syst Rev . 2012;6(6):CD000259. doi:10.1002/14651858.CD000259.pub3

2. MacLean CH, Kerr EA, Qaseem A. Time Out - Charting a Path for Improving Performance Measurement. N Engl J Med . 2018:1-4. doi:10.1056/NEJMp1002530

3. Brehaut JC, Colquhoun HL, Eva KW, et al. Practice Feedback Interventions: 15 Suggestions for Optimizing Effectiveness. Ann Intern Med . 2016;164(6):435-441. doi:10.7326/M15-2248

4. MacKenzie C, Chan TM, Mondoux S. Clinical Improvement Interventions for Residents and Practicing Physicians: A Scoping Review of Coaching and Mentoring for Practice Improvement. AEM Educ Train . 2019;3(4):353-364. doi:10.1002/aet2.10345

5. Sargeant J, Lockyer J, Mann K, et al. Facilitated Reflective Performance Feedback: Developing an Evidence- and Theory-Based Model That Builds Relationship, Explores Reactions and Content, and Coaches for Performance Change (R2C2). Acad Med . 2015;90(12):1698-1706. doi:10.1097/ACM.0000000000000809

6. Sargeant J, Mann K, Manos S, et al. R2C2 in Action: Testing an Evidence-Based Model to Facilitate Feedback and Coaching in Residency.J Grad Med Educ . 2017;9(2):165-170. doi:10.4300/JGME-D-1600398.1

7. Cooke LJ, Duncan D, Rivera L, Dowling SK, Symonds C, Armson H. The Calgary Audit and Feedback Framework: a practical, evidence-informed approach for the design and implementation of socially constructed learning interventions using audit and group feedback. Implement Sci . 2018;13(1):136. doi:10.1186/s13012-018-0829-3

8. Cooke LJ, Duncan D, Rivera L, Dowling SK, Symonds C, Armson H. How do physicians behave when they participate in audit and feedback activities in a group with their peers? Implement Sci . 2018;13(1):1-9. doi:10.1186/s13012-018-0796-8

9. Charmaz K. Constructing Grounded Theory: A Practical Guide Through Qualitative Analysis . 2nd ed. SAGE Publications, Inc.; 2006. 
10. Gupta R, Mercuri M, McCulloch A, et al. Regional needs assessment for emergency physician audit and feedback. CJEM . May 2020:1-7. doi:10.1017/cem.2020.348

11. Dweck CS. Mindset: The New Psychology of Success . New York, New York: Ballantine Books; 2007.

12. Kegan R, Laskow Lahey L. Immunity to Change: How to Overcome It and Unlock the Potential in Yourself and Your Organization. 1st Editio. Harvard Business Review Press; 2009. doi:10.1017/CBO9781107415324.004

13. Kegan R, Lahey LL. An Everyone Culture: Becoming a Deliberately Developmental Organization . Harvard Business Review Press; 2016.

14. Gude WT, Brown B, van der Veer SN, et al. Clinical performance comparators in audit and feedback: a review of theory and evidence.Implement Sci . 2019;14(1):39. doi:10.1186/s13012-019-0887-1

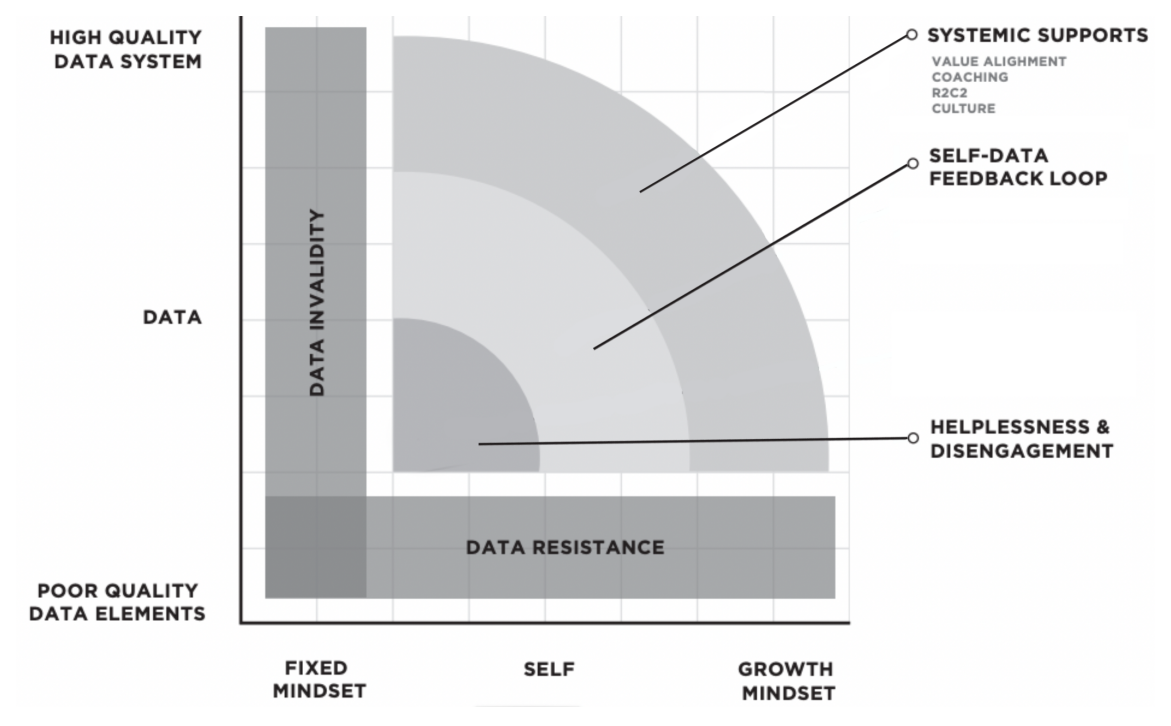

Figure 1: MD-PIE model - A proposed model describing the interactions between data systems and practitioner mindset in producing engagement.

\begin{tabular}{lll}
\hline Demographic & Value & Frequency \\
Gender & M F & 96 \\
Type of practice & Academic Community Mixed & 762 \\
Years in practice & $0-56-1011-1516+$ & 8115 \\
\hline
\end{tabular}

Table 1: demographic features of interview participants. $\mathrm{N}=15$ 


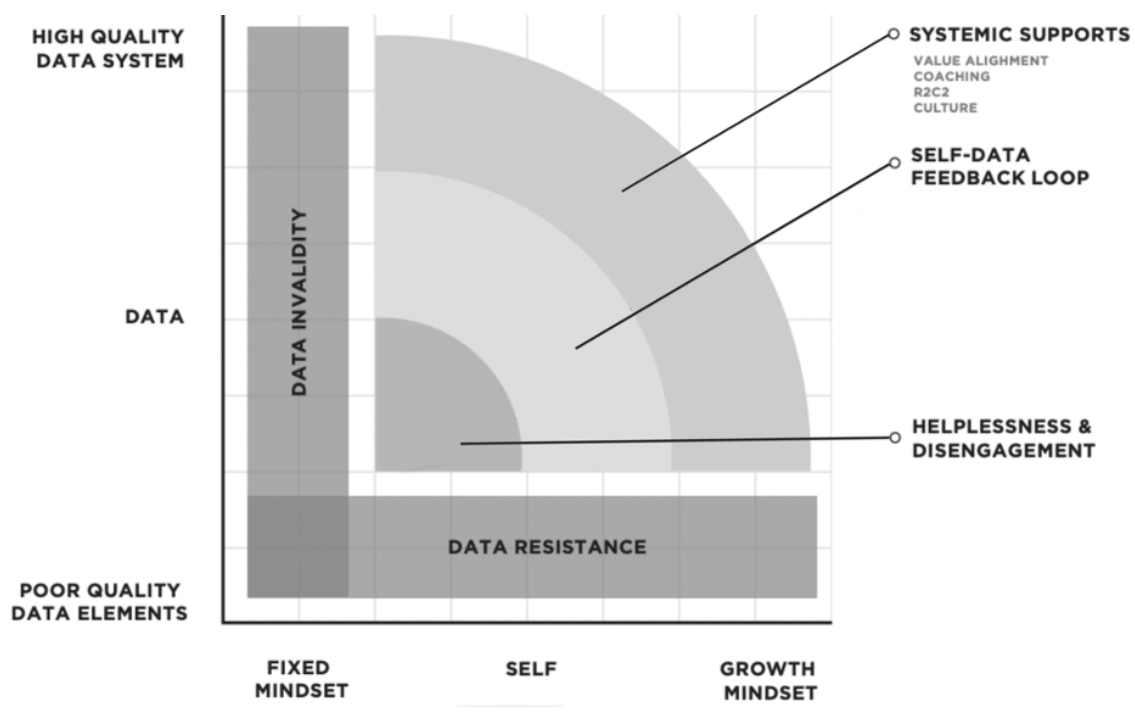

